\title{
Characteristics of Printed Thin Films Using Indium Tin Oxide (ITO) Ink
}

\author{
Sung-Jei Hong ${ }^{1, *}$, Jong-Woong Kim ${ }^{1}$, Jae-Won Lim² ${ }^{2}$, Good-Sun Choi ${ }^{2}$ and Minoru Isshiki ${ }^{3}$ \\ ${ }^{1}$ Display Components and Materials Research Center, Korea Electronics Technology Institute, Seongnam 463-816, Korea \\ ${ }^{2}$ Mineral Resources Research Division, Korea Institute of Geoscience \& Mineral Resources, Deajeon 305-350, Korea \\ ${ }^{3}$ Institute of Multidisciplinary Research for Advanced Materials, Tohoku University, Sendai 980-8577, Japan
}

\begin{abstract}
Characteristics of the indium tin oxide (ITO) thin films fabricated by a direct printing method were investigated. For the direct printing, 30 mass\% ITO ink was formulated by dispersing ITO nanoparticles of less than $10 \mathrm{~nm}$ in diameter into alcohol based solvent with additives. Then, the ITO ink was printed onto a glass substrate, which was followed by the heat treatment at $350,400,500$ and $600^{\circ} \mathrm{C}$ to fabricate the sintered films. Then, cross-section of sintered film was observed. As a result, the ITO thin film showed porous nanostructure. Also, sheet resistance of the ITO thin film decreased with increasing heating temperature. The decrease of the resistance was attributed to the improvement of carrier concentration in the ITO thin film. In the meanwhile, the optical transmittance increased in proportional to the heating temperature. In case of the ITO thin film heated at $600^{\circ} \mathrm{C}$, the improved characteristics of the electrical resistance and the optical transmittance were achieved as $2.19 \times 10^{3} \Omega / \mathrm{sq}$ and $78.6 \%$, respectively. [doi:10.2320/matertrans.MAW201017]
\end{abstract}

(Received April 27, 2010; Accepted July 14, 2010; Published August 25, 2010)

Keywords: indium tin oxide (ITO) Ink, direct printing, electrical resistance, optical transmittance, carrier concentration

\section{Introduction}

Transparent electrode is one of the essential parts of flat panel displays such as touch panel, liquid crystal display (LCD) and organic light emission display (OLED), etc. Also, in recent, applications of the transparent electrode are extended to the industries related to renewable energies such as solar cell. ${ }^{1)}$ As a material of the transparent electrode, indium tin oxide (ITO) has been widely used because of its unique characteristics of optical transparency and electrical conduction. ITO thin film patterns are conventionally made by vacuum processes such as sputtering or evaporation followed by photolithography and chemical etching. However, the vacuum processes have disadvantages of inefficiency because ITO is exhausted onto inner wall of the vacuum chamber as well as coated onto substrate. Especially, the exhaust of indium (In) is being raised as a serious global issue, and the exhaust is accelerated with rapid market growth of the related industries. Thus, price of In increases in proportional to the market growth, and the reduction of In consumption is extensively required. As one of the methods for the reduction, direct printing using ITO ink is paid attention. ${ }^{2)}$ Direct printing is to draw the electrode onto substrate without vacuum processes, photolithography and wet etching. In order to reduce the In consumption effectively, lower concentration of the ITO in the ink and thinner thickness are important factors. However, in a previous study, the values for the concentration of the ITO and the thickness are much larger than 50 mass $\%{ }^{3)}$ In order to reduce In consumption effectively, the concentration and the thickness should be reduced. Therefore, we tried to fabricate the ITO thin films by direct printing with ITO ink with a solid content less than 30 mass $\%$. The ITO films were used to observe the effects of heating conditions on the characteristics of the films.

*Corresponding author, E-mail: hongsj@keti.re.kr

\section{Experimental Procedure}

ITO nanoparticles were prepared by low temperature synthetic method. Detailed process is described in the literature. ${ }^{4)}$ The synthesized ITO nanoparticles were mixed with the alcohol based solvent including dispersing agents to formulate ITO inks. That is, alcohol and ethylene glycol were mixed together, and small amount of polymeric dispersing agent was added to the mixed solvent. Then, ITO nanoparticle was put into the solvent, and was dispersed by mechanical force until the submerged ITO nanoparticle disappeared. Concentration of the ITO nanoparticles in the ink was controlled to be 30 mass $\%$, and the ITO nanoparticle was well dispersed. For determining the lowest temperature to burn out the organic components out of inks except In and $\mathrm{Sn}$, thermal behaviors of the formulated inks were analyzed by using Thermo Gravimetry/Differential Thermal Analyzer (Seiko Exstar 6000, TG/DTA6100). Then, the inks were directly printed by the inkjet printing on a glass substrate via a nozzle of $60 \mu \mathrm{m}$ in hole diameter. After printing, the ITO thin film was heated at $350,400,500$ and $600^{\circ} \mathrm{C}$ for $1 \mathrm{~h}$ in the atmospheric environment. Thickness of the ITO thin film was measured by observing cross-section of the ITO thin film. The characteristics of the ITO thin films were evaluated by observing optical and electrical properties with UV-VIS Spectrophotometer (JASCO, V-560) and 4point probe electrical measurement system (Mitsubishi Chemical Analytech, MCP-T610), respectively. Hall effect measurement system (Ecopia, HMS-3000) was also employed to evaluate the carrier concentration in a magnetic field of $0.55 \mathrm{~T}$ at room temperature using van der Pauw method.

\section{Results and Discussion}

ITO nanoparticles were uniformly dispersed as shown in Fig. 1. That is, aggregation between ITO nanoparticles was minimized. In general, nanoparticles are apt to aggregate 


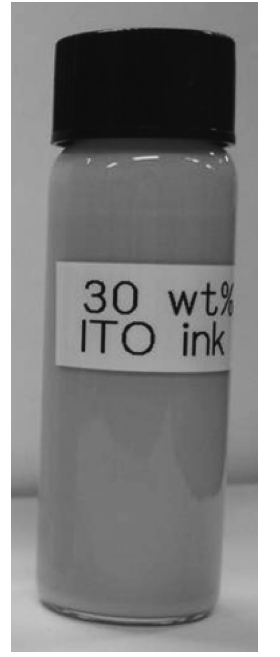

Fig. 1 ITO ink formulated with concentration of 30 mass $\%$.

together for decreasing unstable surface area. The aggregation is attributed to the attractive interactions between the particles to reduce the surface energy. The surface area of a nanoparticle is about $10^{5}$ times to that of a microparticle. Thus, the driving force to decrease the surface area is very high that the aggregation of the nanoparticles is spontaneously occurred. Therefore, to disperse the nanoparticle, external potential energy to minimize the aggregation was applied by using organic additives. In Derjiaguin, Landau, Verwey and Overbeek (DLVO) theory, the potential energies of attraction and repulsion are summed to provide a total interaction potential energy between colloidal particles. ${ }^{5)}$ The total interaction potential energy $(\Delta E)$ can be expressed as

$$
\Delta E=-E_{\mathrm{A}}+E_{\mathrm{R}}
$$

where $E_{\mathrm{A}}$ and $E_{\mathrm{R}}$ are interaction potential energies of attraction and repulsion, respectively. The interaction potential energies of attraction and repulsion are generated by van der Waals force and electrostatic force, respectively. In case of nano-dimensional particle, $E_{\mathrm{A}}$ is much larger than $E_{\mathrm{R}}$ because of their low electrostatic charge, which is the surface charge density times the surface area. ${ }^{6}$ Accordingly, $\Delta E$ shows a negative value, and the ITO nanoparticle aggregates. Therefore, in order to disperse the ultrafine ITO nanoparticle uniformly, the $\Delta E$ should have a positive value by increasing the $E_{\mathrm{R}}$. Polymer based additives were supplied into solution in order to generate the electrostatic repulsive force by surrounding the ITO nanoparticles to separate them. From the DLVO theory, it is guessed that the $\Delta E$ goes to the negative value as the size of ITO nanoparticle decreases leading to uniform dispersion.

As well, thermal behavior of ITO ink revealed that its heating temperature is able to be controlled starting from $250^{\circ} \mathrm{C}$. As seen in Fig. 2, TGA analysis showed a steep decrease in weight of ITO ink to 22 mass $\%$ at $160^{\circ} \mathrm{C}$, and an additional slight change in weight to 30 mass $\%$ was observed at $250^{\circ} \mathrm{C}$. At similar temperature, the change in heat flow was observed. In addition, slight changes in heat flow were observed at $350^{\circ} \mathrm{C}$. Those phenomena are owing to the thermal decomposition of the polymeric components out of the ink. That is, the solvent and additives which are consisted

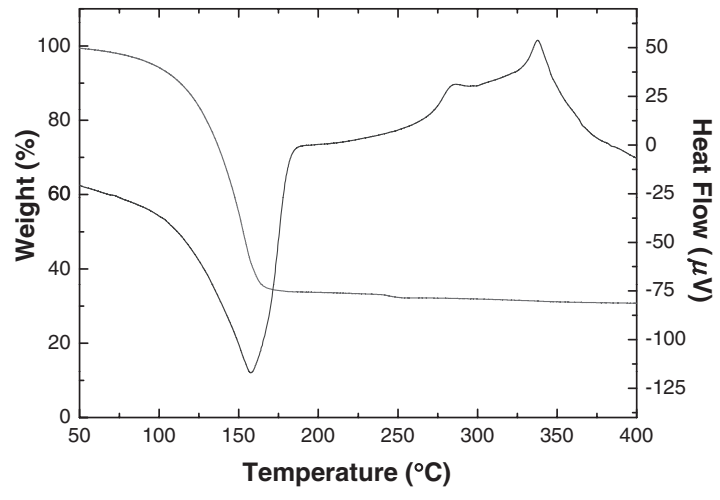

Fig. 2 Thermal behavior of formulated ITO ink.

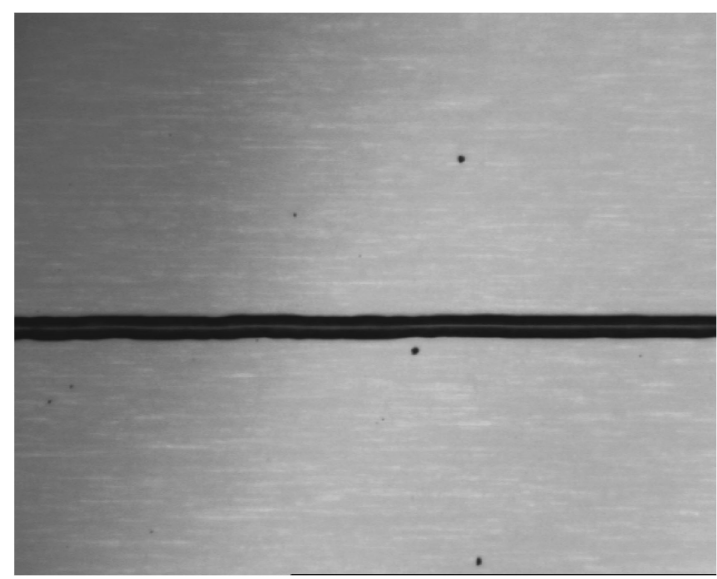

(a)

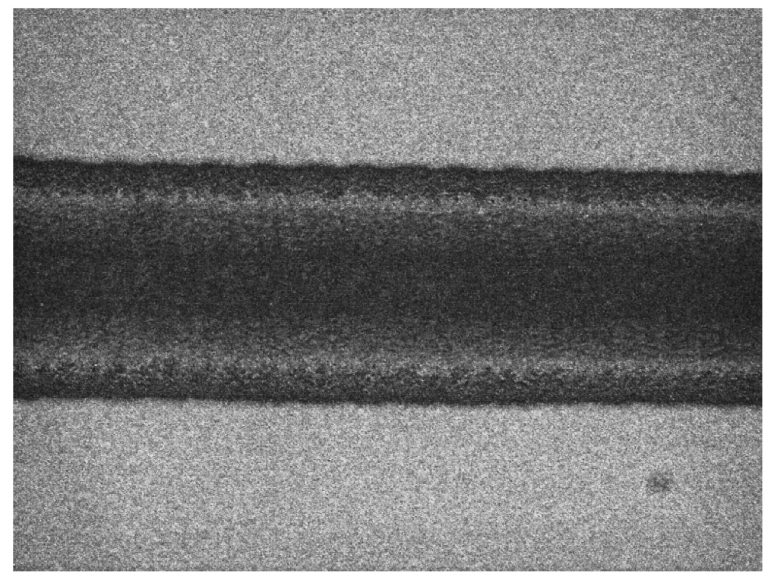

(b)

Fig. 3 ITO thin film fabricated by inkjet printing (a) Overview (b) Magnified view.

of polymeric components are burnt out of ink to leave mere ITO nanoparticle.

ITO thin film pattern was printed using the formulated ITO ink, and the pattern was well printed as shown in Fig. 3. This is owing to the uniform dispersion of the ITO nanoparticles in the ink. That is, the dispersion of the ITO nanoparticles was maintained until passing through the micro-nozzle of an inkjet printing head. As discussed above, polymeric additives are seemed to help the dispersion of ITO nanoparticle. As 


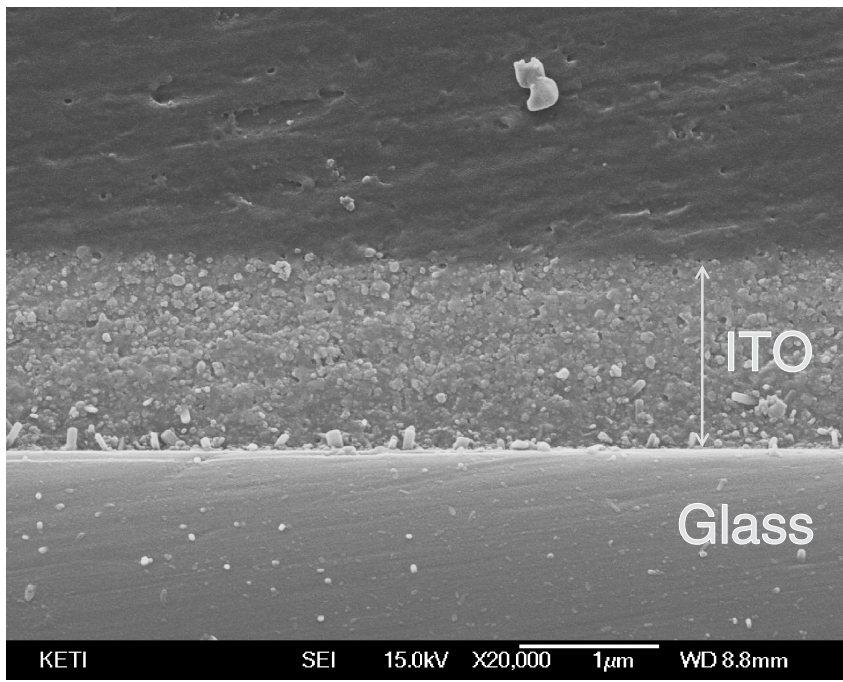

Fig. 4 Cross-sectional nanostructure of ITO thin film.

well, the ITO thin film printed on a glass substrate showed transparent properties by naked eyes after heat-treatment at above $350^{\circ} \mathrm{C}$. This is owing to the thermal decomposition of the polymeric additives at the temperature range. That is, as discussed, this seems to be related to the slight change in heat flow at $350^{\circ} \mathrm{C}$. It is guessed that the residual polymeric additives were burnt out at the temperature. Thus, the printed ITO thin film was heated at above $350^{\circ} \mathrm{C}$.

After the heat-treatment, cross-section of ITO thin film was observed. As a result, shown in Fig. 4, the thickness was about $1.29 \mu \mathrm{m}$. As well, some of pores in the ITO thin film were observed. The pores are assumed to be attributed to heat-treatment condition. That is, the heat-treatment condition has to be optimized in order that ITO nanoparticles have to coalesce uniformly as organic components are thermally decomposed to evaporate out of the thin film. However, the heat-treatment condition under atmosphere environment was not enough to approach the ideal state because those defects like pores were generated. Such defects are afraid to affect the electrical and optical properties. In order to improve the defect structure, optimal heat-treatment condition has to be investigated.

Then, the electrical and optical properties of the printed ITO thin film were characterized. In case of electrical properties, shown in Fig. 5, sheet resistance of the ITO thin film decreased with the raising heating temperature. That is, sheet resistance of the ITO thin films heated at 350, 400, 500 and $600^{\circ} \mathrm{C}$ were $24.20 \times 10^{3}, 4.81 \times 10^{3}, 2.94 \times 10^{3}$ and $2.19 \times 10^{3} \Omega / \mathrm{sq}$, respectively. This is attributed to the improvement of microstructure of the ITO thin film. In general, electrical carriers go through the thin film by way of microstructure. ${ }^{7)}$ Also, defects such as vacancy existing in the microstructure act as trapping sites to lower the mobility of the electrical carriers. Therefore, the microstructure was seemed to be improved by the raising heating temperature leading to an increase of carrier concentration.

In order to investigate those phenomena, carrier concentration of the printed ITO thin films was measured. As a result, shown in Fig. 6(a), Hall mobility was enhanced by the raising heating temperature. That is, Hall mobility of the

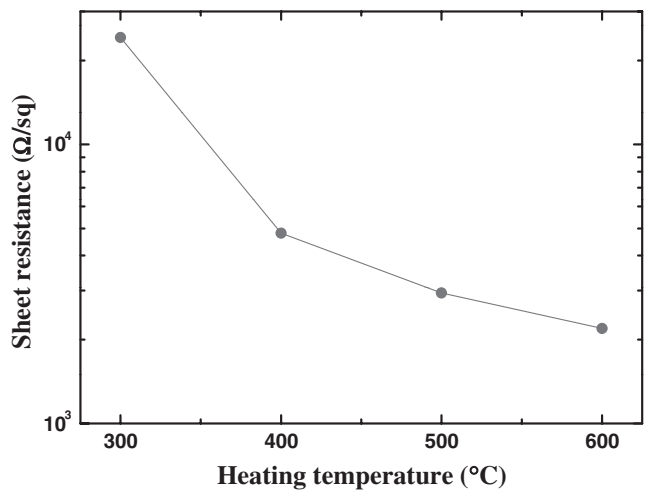

Fig. 5 Electrical properties of printed ITO thin films changed with heating temperature.

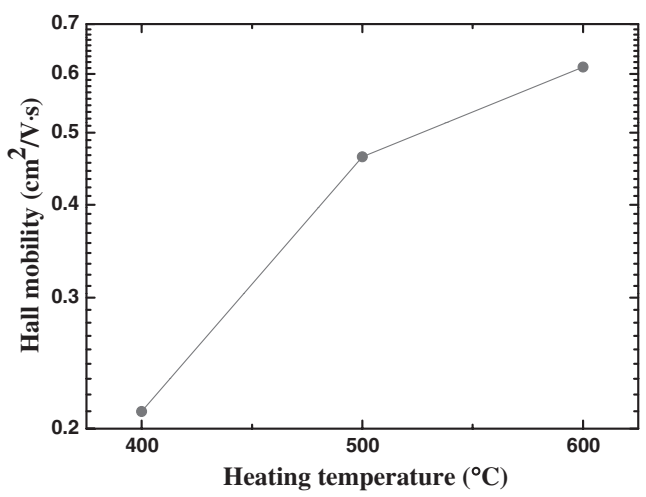

(a)

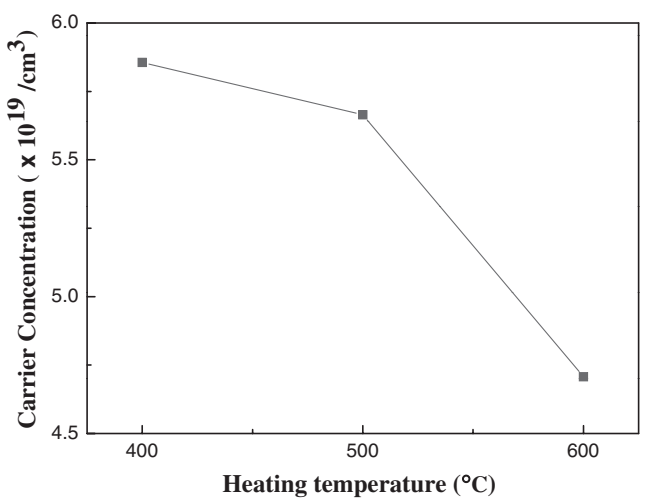

(b)

Fig. 6 Hall mobility and carrier concentration of printed ITO thin changed with heating temperature. (a) Hall mobility (b) Carrier concentration.

printed ITO thin films followed by heat-treatment at 400, 500 and $600^{\circ} \mathrm{C}$ was $0.211,0.464$ and $0.613 \mathrm{~cm}^{2} / \mathrm{V} \cdot \mathrm{s}$, respectively. Hall mobility of the ITO thin film followed by heat-treatment at $350^{\circ} \mathrm{C}$ was not measured. From this result, we assume that the enhancement of the electrical properties of the printed ITO thin film with increase of heating temperature is owing to the improvement of the Hall mobility. As shown in Fig. 6(b), carrier concentration was decreased despite of increase of heating temperature. That is, carrier concentration of ITO thin film followed by heat-treatment at 400, 500 and $600^{\circ} \mathrm{C}$ was $5.86 \times 10^{19}, 5.66 \times 10^{19}$ and $4.71 \times 10^{19} / \mathrm{cm}^{3}$, respectively. It is reported that carrier concentration is decreased in spite of increase of Hall mobility, and such a 


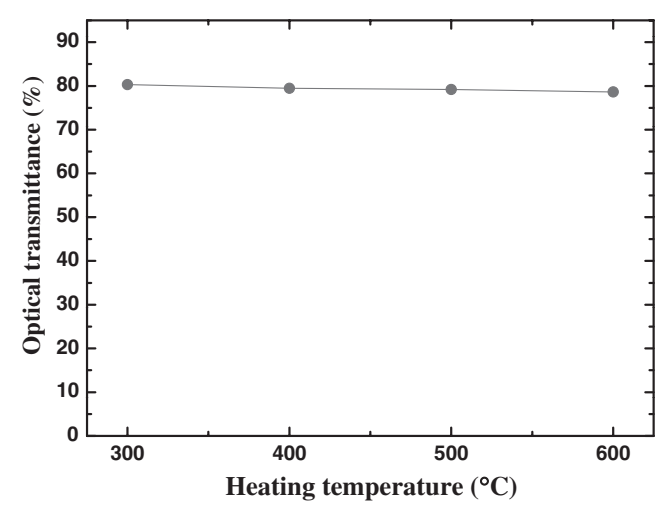

Fig. 7 Optical properties of printed ITO thin films changed with heating temperature.

behavior is owing to impurity scattering in the highly crystallized lattice structure of ITO crystal. ${ }^{7)}$ Thus, in this case, it is supposed that the impurity scattering mechanism is dominant. The values carrier concentrations of ITO thin films fabricated by inkjet printing is lower than those by conventional method under vacuum condition. ${ }^{8,9}$ In order to improve the carrier concentration, researches are being continued.

In case of optical properties, transmittance of the printed ITO thin film slightly decreased with increasing heating temperature. As shown in Fig. 7, at $600 \mathrm{~nm}$ of the bandwidth which is the central range of the visible light, optical transmittance of the ITO thin films heated at 350, 400, 500 and $600^{\circ} \mathrm{C}$ was $80.3,79.5,79.2$ and $78.6 \%$, respectively. This should be concerned with increase of electrical carrier in the ITO thin film. That is, as the concentration of the electrical carriers increases, the carriers act as an attenuation center to deteriorate the optical transmittance.

However, the values of electrical resistance and optical transmittance are not satisfactory compared to those fabricated by conventional method under vacuum condition. ${ }^{8,9)}$ In order to improve those optical properties with the improvement of the electrical properties, studies are being continued with the investigation on other parameters.

\section{Conclusions}

ITO thin film pattern was well fabricated by inkjet printing with ITO ink. The ITO ink was well formulated with uniform dispersion of 30 mass \% ITO nanoparticle into alcohol based solvent with additives. The ITO ink was printed onto a glass substrate without any problem for the fabrication of the ITO thin film by inkjet printing. Sheet resistance of the printed ITO thin film decreased with increasing heating temperature. The decrease of resistance was attributed to the improvement of the carrier concentration in the ITO thin film. In the meantime, the optical transmittance increased in proportional to the heating temperature. In case of the ITO thin films heated at $600^{\circ} \mathrm{C}$, the improved characteristics of the electrical resistance and the optical transmittance were achieved as $2.19 \times 10^{3} \Omega /$ sq and $78.6 \%$, respectively.

\section{Acknowledgements}

This work was supported by the Energy \& Resource Recycling of the Korea Institute of Energy Technology Evaluation and Planning (KETEP) grant funded by the Korea government Ministry of Knowledge Economy (No. 2008K00202).

\section{REFERENCES}

1) T. Minami: Thin Solid Films 516 (2008) 1314-1321.

2) S. Heusing, P. W. de Oliveira, E. Kraker, A. Haase, C. Palfinger and M. Veith: Thin Solid Films 518 (2009) 1164-1169.

3) J. Puetz and M. A. Aegerter: Thin Solid Films 516 (2008) 4495-4501.

4) S. J. Hong, Y. H. Kim and J. I. Han: IEEE Trans. Nanotech. 7 (2008) $172-176$.

5) J. Sun, B. V. Velamakanni, W. W. Gerberich and L. F. Francis: J. Col. Int. Sci. 280 (2004) 387-399.

6) S. N. Kallay and S. Zalac: J. Col. Int. Sci. 253 (2002) 70-76.

7) H.-C. Lee: Appl. Surf. Sci. 252 (2006) 2647-2656.

8) H. Ohta, M. Orita, M. Hirano, H. Tanji, H. Kawazoe and H. Hosono: Appl. Phys. Lett. 76 (2000) 2740-2742.

9) Y. Sawada, C. Kobayashi, S. Seki and H. Funakubo: Thin Solid Films 409 (2002) 46-50. 